\title{
Estado del Mobile Learning en España
}

\section{Estado de Mobile Learning na Espanha}

\section{Situation of Mobile Learning in Spain}

\author{
Francisco Brazuelo Grund ${ }^{1}$ \\ Domingo José Gallego Gil ${ }^{1}$
}

\begin{abstract}
RESUMEN
Las tecnologías móviles se han convertido en artefactos omnipresentes en la vida diaria de las personas. Este hecho ha propiciado un especial interés por explorar y explotar estos medios como potenciales recursos didácticos, dando lugar al Mobile Learning o Aprendizaje Móvil. Esta modalidad educativa, aunque reciente, se haya extendida en todo el mundo, especialmente en instituciones educativas y en contextos de formación de organizaciones y empresas. El presente artículo expone una investigación sobre el estado del Mobile Learning o Aprendizaje Móvil acotado a España a partir de un estudio de revisión descriptivo de la producción científica nacional en el período que abarca desde principios del 2009 hasta finales del 2013, y cuyos resultados muestran un creciente interés nacional por el establecimiento de bases teóricas y la experimentación para integración de las tecnologías móviles en educación.

Palabras clave: mobile learning; e-learning; producción científica; España.
\end{abstract}

\section{RESUMO}

As tecnologias móveis tornaram-se dispositivos onipresentes na vida cotidiana das pessoas. Isto levou a um interesse especial na exploração e aproveitamento dos meios de comunicação como potenciais recursos de ensino, levando à aprendizagem móvel ou Mobile Learning. Esta, apesar de recente modalidade educacional, tem sido difundida em todo o mundo, especialmente em instituições de ensino e contextos de formação de organizações e empresas. Este artigo apresenta uma investigação sobre o estado do Mobile Learning ou aprendizagem móvel limitada à Espanha a partir de

DOI: $10.1590 / 0104-4060.38646$

1 Universidad Nacional de Educación a Distancia - UNED - Facultad de Educación. Madrid, España. Calle Juan del Rosal, 14, 28040. 
uma revisão de estudo descritivo da produção científica nacional, no período que vai do início de 2009 até o final de 2013, cujos resultados mostram um crescente interesse nacional, estabelecendo e testando bases teóricas para a integração de tecnologias móveis na educação.

Palavras-chaves: mobile learning; e-learning; produção científica; Espanha.

\begin{abstract}
Mobile technologies have become ubiquitous devices in daily life of people. This has led to a special interest in exploring and exploiting the media as potential teaching resources, leading to the Mobile Learning. Although Mobile Learning is a new educational system, it has been widespread throughout the world, especially in educational institutions and training contexts of organizations and companies. This paper presents an investigation into the status of Mobile Learning bounded in Spain from a descriptive study review of national scientific production in the period from early 2009 to late 2013. The results show an increasing national interest by establishing and testing theoretical basis for integration of mobile technologies in education.

Keywords: mobile learning; e-learning; scientific production; Spain.
\end{abstract}

\title{
Introducción
}

El Mobile Learning o Aprendizaje Móvil, como tema de investigación en tecnologías de la educación, se inicia aproximadamente a principios de la primera década de este siglo. La principal característica del Mobile Learning o Aprendizaje Móvil es la ubicuidad, es decir, permite el desarrollo del proceso de enseñanza y aprendizaje en cualquier momento y lugar. En principio podría asociarse a cualquier tecnología móvil pero en el campo educativo destacan tres: los smartphones o teléfonos móviles inteligentes, los tablets o tabletas digitales y los phablets, dispositivo resultado de la hibridación de los dos primeros anteriormente mencionados.

Hasta su actual estado de consolidación ha estado en continua evolución destacándose tres fases (PACHLER; BACHMAIR; COOK, 2010): la primera fase, tecnocéntrica, evolutiva a partir del eLearning, y centrada en la persona y su contexto de aprendizaje. 
En sus inicios el Aprendizaje Móvil estaba mediatizado por la tecnología en sí y se había definido como la mera utilización de dispositivos electrónicos portables para la modificación de conductas (O'MALLEY y otros, 2005; KEEGAN, 2005). Más adelante, en una segunda fase, se considera como un continuo del e-Learning (similar a un e-Learning pero en "miniatura") aunque se incorpora el concepto de ubicuidad y se potencia el concepto de flexibilidad del aprendizaje (GEORGIEV; GEORGIEVA; TRAJOVSKI, 2006).

Sin embargo, las tendencias anteriores dejaban de lado dos aspectos cruciales para el actual concepto de Mobile Learning o Aprendizaje Móvil y que aparecen en la tercera fase evolutiva: la movilidad y, en consecuencia, la variabilidad del contexto de aprendizaje.

En el caso educativo, es el estudiante quien se mueve y con él cualquier tecnología móvil que lleve consigo. Debe considerarse a ésta no como un fin, sino sólo un medio facilitador de oportunidades de aprendizaje, especialmente cuando existe movimiento físico. Porque al moverse, cambia el contexto de aprendizaje. Hasta ahora se había asumido que el aprendizaje formal tenía lugar en un aula y con mediación de un profesor, es decir, un contexto cerrado y mediatizado, sin tener en cuenta el factor de movilidad. Este factor hace variable el contexto y hace fluir el aprendizaje a través de diversas localizaciones a través del tiempo que posteriormente se puede transferir a otros contextos completamente distintos.

Para establecer una definición de Mobile Learning o Aprendizaje Móvil, no pueden obviarse tres conceptos clave: tecnologías móviles; ubicuidad vinculada a la movilidad; y usos educativos en contextos variables. La Organización de las Naciones Unidas para la Educación, la Ciencia y la Cultura (UNESCO) propone, en este sentido, la siguiente definición que "El aprendizaje móvil comporta la utilización de tecnología móvil, sola o en combinación con cualquier otro tipo de Tecnología de la Información y la Comunicación (TIC), a fin de facilitar el aprendizaje en cualquier momento y lugar" (UNESCO 2013, p. 6).

Finalmente, y haciendo un ejercicio de síntesis de las distintas concepciones sobre qué es el Mobile Learning o Aprendizaje Móvil, se propone una definición que condensa el concepto y alcance de este término como "[...] modalidad educativa que facilita la construcción del conocimiento, la resolución de problemas de aprendizaje y el desarrollo de destrezas o habilidades diversas de forma autónoma y ubicua gracias a la mediación de dispositivos móviles portables" (BRAZUELO; GALLEGO, 2011, p. 17). 


\section{Objetivos}

Los objetivos de la investigación son los siguientes:

1. Localizar la literatura científica española sobre el Mobile Learning o Aprendizaje Móvil en el período de principios del 2009 hasta finales del 2013;

2. Analizar la producción científica detectada en España sobre el Mobile Learning o Aprendizaje Móvil en el período mencionado;

3. Identificar los núcleos temáticos de investigación y experimentación de la temática de estudio en el ámbito nacional español;

4. Acercar a la comunidad científica literatura de referencia acerca del Mobile Learning o Aprendizaje Móvil en España;

5. Diagnosticar el estado del Mobile Learning en España.

\section{Metodología}

Para alcanzar los objetivos propuestos en esta investigación bibliográfica hemos realizado una revisión, de carácter descriptivo, de la producción científica sobre Mobile Learning o Aprendizaje Móvil en el período que abarca desde inicios del 2009 hasta finales del 2013 en España, que ofrecemos detalladamente en el Anexo de este trabajo.

La recogida de datos tuvo lugar a finales de 2013. El universo de estudio ha sido extraído de fuentes primarias y secundarias, prioritariamente indexadas en bases de datos nacionales, con relación al objeto de la investigación (DAY, 2005; GUIRAO-GORIS; OLMEDO; FERRER, 2008): tesis doctorales, libros, eBooks, capítulos de libros, artículos de revistas científicas y artículos de actas de congresos, seminarios y jornadas.

La estrategia metodológica se inicia con la localización de los textos científicos. La búsqueda se realiza en títulos y resúmenes o abstracts de los documentos a partir de palabras clave. Con el fin de ampliar al máximo el espectro de búsqueda, se crean tres grupos de palabras clave relacionadas con la etiqueta "Mobile Learning", con las tecnologías móviles y las funcionalidades de los dispositivos móviles, a saber:

- GRUPO 1: Mobile Learning, Aprendizaje Móvil, MLearning, M-Learning, Aprendizaje Ubicuo, uLearning y U-Learning; 
- GRUPO 2: teléfono móvil, smartphone, phablet, tablet, tabletas, iPad, dispositivos móviles y tecnologías móviles;

- GRUPO 3: SMS, Realidad Aumentada, App, Aplicaciones Móviles, podcast, Códigos QR, multimedia móvil, Android, iOS.

Para estrechar las búsquedas con las palabras clave de los grupos 2 y 3 , cada una se combinó con una de las siguientes: educación, education, aprendizaje, learning, clase, classroom.

Tras la localización de los textos se adopta como criterio de inclusión documentos directamente relacionados con la temática de estudio de la investigación y, prioritariamente, publicados en bases de datos, revistas científicas y actas de congresos y otros eventos científicos.

Las tesis doctorales fueron extraídas de TESEO, base de datos de Tesis Doctorales del Ministerio de Educación, Cultura y Deporte de España, accesible en $<$ https://www.educacion.gob.es/teseo $>$.

Para los libros y capítulos de libro se recurre a la base de datos de libros editados en España (<http://www.mcu.es/libro/CE/AgenciaISBN/BBDDLibros/ Sobre.html $>$ ).

Los artículos de revistas científicas y actas de eventos científicos son localizados a través de las bases de datos (Dialnet, Redined, ISOC-Ciencias Sociales, REDALYC, IN-RECS); accediendo directamente a las fuentes, en base al listado proporcionado por DICE (entidad par la Difusión y Calidad Editorial de las Revistas Españolas de Humanidades y Ciencias Sociales); y realizando consultas en buscadores como Tesauro, Academica EDU y Google Scholar.

\section{Análisis de resultados}

El total de documentos localizados, y que cumplen los criterios de inclusión anteriormente mencionados se cifra en 190 (Anexo I). La Figura 1 representa la evolución de la producción científica sobre Mobile Learning o Aprendizaje Móvil en el período 2009-2013 en España.

De esta producción científica, $2(1 \%)$ documentos corresponden a tesis doctorales; 5 a libros o eBooks (2,5\%); 21 a capítulos de libros (11\%); 85 a artículos de revistas (44,5\%) y 77 a artículos de eventos científicos (41\%), principalmente actas de congresos (Figura 2). Como podemos contrastar, el 85,5\% corresponden a artículos de revistas y actos científicos. 


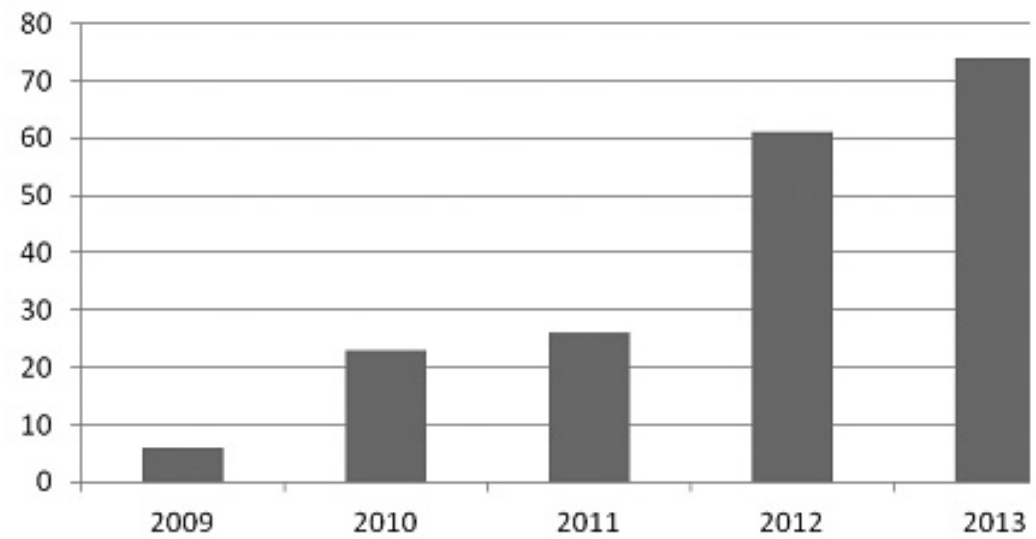

FIGURA 1 - PRODUCCIÓN CIENTÍFICA TOTAL SOBRE MOBILE LEARNING O APRENDIZAJE MÓVIL EN ESPAÑA (2009-2013)

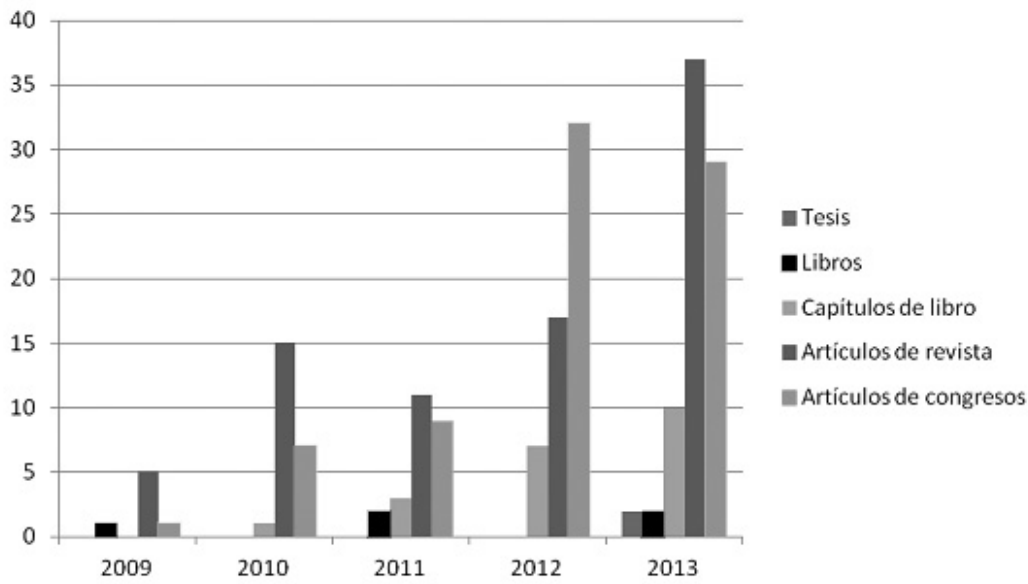

FIGURA 2 - PRODUCCIÓN CIENTÍFICA DETALLADA SOBRE MOBILE LEARNING O APRENDIZAJE MÓVIL EN ESPAÑA (2009-2013)

Las revistas con aportaciones se presentan en el siguiente cuadro ordenadas por número de documentos publicados por año de mayor a menor (Tabla 1). En total han sido examinadas 49 revistas, 14 de las cuales no han realizado publicaciones relacionadas con el tema de nuestra investigación (Revista Informática Educativa y Comunicaciones; Quaderns Digitals; REDU Revista de Docencia 
Universitaria; Educatio Siglo XXI; Revista de Formación e Innovación Educativa Universitaria; REDEX. Revista de Educación de Extremadura; Enseñanza y Teaching; Revista de Educación MEC; Revista Electrónica Interuniversitaria de Formación del Profesorado; Revista Electrónica de Investigación y Docenci; Revista Educación y Futuro Digital; Red digital; Bordón. Revista de Pedagogía).

TABLA 1 - PRODUCCIÓN CIENTÍFICA EN TORNO AL MOBILE LEARNING EN REVISTAS ESPAÑOLAS (2009-2013)

\begin{tabular}{|c|c|c|c|c|c|c|}
\hline Revistas/Período de publicación & 2009 & 2010 & 2011 & 2012 & 2013 & $\mathbf{T}$ \\
\hline RED. Revista de Educación a Distancia & 0 & 1 & 0 & 2 & 6 & 9 \\
\hline Didáctica, Innovación y Multimedia (DIM) & 0 & 0 & 0 & 3 & 3 & 6 \\
\hline Eufonía. Didáctica de la Música & 0 & 0 & 0 & 2 & 3 & 5 \\
\hline Comunicación y Pedagogía & 0 & 0 & 0 & 5 & 0 & 5 \\
\hline Teoría de la Educación & 0 & 1 & 1 & 0 & 3 & 5 \\
\hline IEEE-Rita & 0 & 4 & 0 & 0 & 0 & 4 \\
\hline Edutec-e & 0 & 1 & 1 & 0 & 2 & 4 \\
\hline RIED: Revista Iberoamericana de Educación a Distancia & 2 & 2 & 0 & 0 & 0 & 4 \\
\hline Pixel-Bit & 0 & 2 & 1 & 1 & 0 & 4 \\
\hline Eduteka & 0 & 0 & 0 & 1 & 3 & 4 \\
\hline AULA de Innovación Educativa & 0 & 0 & 0 & 0 & 3 & 3 \\
\hline Etic@net & 0 & 0 & 2 & 0 & 1 & 3 \\
\hline Comunicar & 1 & 1 & 0 & 0 & 0 & 2 \\
\hline Digital Education Review & 0 & 0 & 0 & 0 & 2 & 2 \\
\hline Revista Universitaria y Sociedad del Conocimiento (RUSC) & 0 & 1 & 1 & 0 & 0 & 2 \\
\hline TELOS. Cuadernos de Comunicación e Innovación & 1 & 1 & 0 & 0 & 0 & 2 \\
\hline Icono14. Revista de Comunicación Audiovisual y NNTT & 0 & 0 & 2 & 0 & 0 & 2 \\
\hline EmásF: Revista Digital de Educación Física & 0 & 0 & 0 & 0 & 2 & 2 \\
\hline @itic. Revista de Innovació Educativa & 0 & 0 & 0 & 1 & 0 & 1 \\
\hline RELATEC: Revista Latinoamericana de Tecnología & 0 & 0 & 0 & 0 & 1 & 1 \\
\hline RELIEVE. Revista Electrónica de Investigación Educativa & 0 & 0 & 0 & 0 & 1 & 1 \\
\hline Revista Didáctica de las Ciencias Experimentales y Sociales & 0 & 0 & 0 & 1 & 0 & 1 \\
\hline Cuadernos de Documentación Multimedia & 1 & 0 & 0 & 0 & 0 & 1 \\
\hline Historia y Comunicación Social & 0 & 0 & 0 & 0 & 1 & 1 \\
\hline Pulso, Revista de Educación & 0 & 1 & 0 & 0 & 0 & 1 \\
\hline Aloma. Revista Ciències de l'Educació i de l'Esport & 0 & 0 & 0 & 0 & 1 & 1 \\
\hline EDMETIC. Revista Mediática y Aplicación de las TIC & 0 & 0 & 0 & 0 & 1 & 1 \\
\hline Epsilon: Revista Andaluza de Matemáticas & 0 & 0 & 0 & 0 & 1 & 1 \\
\hline Edetania & 0 & 0 & 0 & 0 & 1 & 1 \\
\hline Revista Iberoamericana de Informática Educativa & 0 & 0 & 0 & 0 & 1 & 1 \\
\hline $\begin{array}{l}\text { Revista de Ciències de L'educació: Universitas } \\
\text { Tarraconensis }\end{array}$ & 0 & 0 & 1 & 0 & 0 & 1 \\
\hline $\begin{array}{l}\text { Novática: Revista de la Asociación de Técnicos de } \\
\text { Informática }\end{array}$ & 0 & 0 & 1 & 0 & 0 & 1 \\
\hline Revista Tendencias Pedagógicas & 0 & 0 & 0 & 0 & 1 & 1 \\
\hline Estudios sobre el Mensaje Periodístico & 0 & 0 & 0 & 1 & 0 & 1 \\
\hline Profesorado: Revista Formación del Profesorado & 0 & 0 & 1 & 0 & 0 & 1 \\
\hline TOTALES ANUALES & 5 & 15 & 11 & 17 & 37 & 85 \\
\hline
\end{tabular}


En cuanto a los artículos procedentes de eventos científicos (congresos, jornadas y seminarios) en relación al Mobile Learning o Aprendizaje Móvil, se han obtenido los siguientes resultados (Tabla 2):

\section{TABLA 2 - PRODUCCIÓN CIENTÍFICA EN TORNO AL MOBILE LEARNING EN EVENTOS CIENTÍFICOS CELEBRADOS EN ESPAÑA (2009-2013)}

\begin{tabular}{|c|c|c|c|c|c|c|}
\hline Actos científicos/Convocatorias & 2009 & 2010 & 2011 & 2012 & 2013 & $\mathbf{T}$ \\
\hline $\begin{array}{l}\text { Congreso sobre Tecnología Educativa y de las Nuevas } \\
\text { Tecnologías aplicadas a la educación (EDUTEC) }\end{array}$ & & 1 & & 6 & & 7 \\
\hline $\begin{array}{l}\text { Congreso Internacional sobre Aprendizaje, Innovación y } \\
\text { Competividad (CINAIC) }\end{array}$ & & & 0 & & 5 & 5 \\
\hline $\begin{array}{l}\text { Congreso Internacional de Tecnologías para la Educación y } \\
\text { el Conocimiento (UNED) }\end{array}$ & 1 & 2 & 4 & 16 & 7 & 30 \\
\hline $\begin{array}{l}\text { Ikasnabar. VI Congreso Internacional de Educación Abierta } \\
\text { y Tecnología (UPV-EHU) }\end{array}$ & & & & & 1 & 1 \\
\hline $\begin{array}{l}\text { TecnoNEET. Congreso Nacional de Tecnología Educativa y } \\
\text { Atención a la Diversidad }\end{array}$ & & 0 & & 0 & & 0 \\
\hline $\begin{array}{l}\text { Congreso Internacional Buenas Prácticas con TIC } \\
\text { (Universidad de Málaga) }\end{array}$ & 0 & & 0 & & 1 & 1 \\
\hline $\begin{array}{l}\text { Congreso Virtual Internacional sobre Innovación } \\
\text { Pedagógica y Praxis Educativa (INNOVAGOGÍA) }\end{array}$ & & & & 1 & & 1 \\
\hline $\begin{array}{l}\text { Congreso Internacional Sociedad Digital: Espacios para la } \\
\text { interactividad y la inmersión }\end{array}$ & & & 2 & & & 2 \\
\hline $\begin{array}{l}\text { Congreso Internacional de Tecnología, Educación y } \\
\text { Desarrollo (INTED de Valencia) }\end{array}$ & & 1 & & & 4 & 5 \\
\hline $\begin{array}{l}\text { Jornadas de Innovación Educativa de la Universidad de la } \\
\text { Laguna }\end{array}$ & & 0 & 0 & 0 & 2 & 2 \\
\hline $\begin{array}{l}\text { Congreso Internacional sobre calidad y accesibilidad de la } \\
\text { Formación Virtual }\end{array}$ & & 0 & & 3 & & 3 \\
\hline $\begin{array}{l}\text { Congreso Internacional Modelos de Investigación Educativa } \\
\text { (AIDIPE) }\end{array}$ & 0 & & & & 3 & 3 \\
\hline $\begin{array}{l}\text { Congreso Internacional de Intercambio de Experiencias de } \\
\text { Innovación Docente Universitaria }\end{array}$ & & & & 1 & & 1 \\
\hline $\begin{array}{l}\text { Congreso Europeo de Tecnologías de la Información en la } \\
\text { Educación y la Sociedad }\end{array}$ & & & & 1 & & 1 \\
\hline Congreso Anual AEDEM & & & & & 2 & 2 \\
\hline $\begin{array}{l}\text { Congreso Internacional de Educación y Nuevas Tecnologías } \\
\text { (EDULEARN de Barcelona) }\end{array}$ & 0 & 0 & 0 & 1 & 0 & 1 \\
\hline $\begin{array}{l}\text { Congreso Internacional sobre Investigación e Innovación en } \\
\text { Educación (ICERI) }\end{array}$ & 0 & 0 & 1 & 1 & 1 & 3 \\
\hline Jornadas de Innovación Educativa (Universidad de Vigo) & & 1 & & & & 1 \\
\hline Congreso Internacional de Gestión del Talento & 0 & & 0 & & 2 & 2 \\
\hline $\begin{array}{l}\text { Jornadas internacionales. Tecnología móvil e innovación en } \\
\text { el aula: nuevos retos y realidades educativas }\end{array}$ & & & & & 3 & 3 \\
\hline $\begin{array}{l}\text { Jornadas eMadrid sobre investigación y el desarrollo de } \\
\text { tecnologías de apoyo al aprendizaje }\end{array}$ & & 2 & 1 & & & 3 \\
\hline TOTALES ANUALES & 1 & 7 & 8 & 30 & 31 & 77 \\
\hline
\end{tabular}


Puede apreciarse la trayectoria, ascendente con los años, de la producción de artículos para revistas y eventos científicos en la siguiente figura (Figura 3), especialmente relevante a partir del 2012:

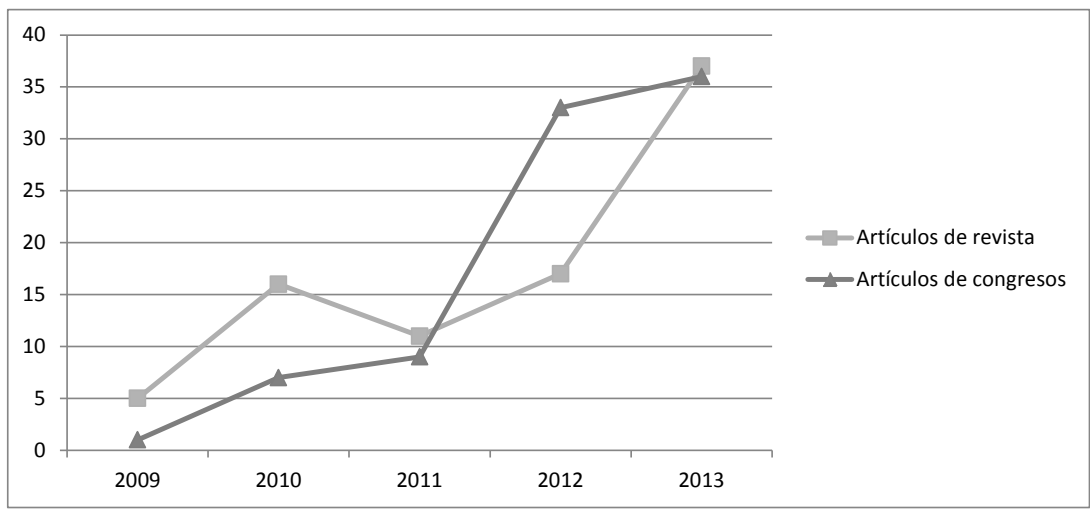

FIGURA 3 - DOCUMENTOS CIENTÍFICOS DE REVISTAS Y ACTAS PUBLICADOS EN ESPAÑA ENTRE AÑOS 2009 AL 2013

En relación a los núcleos temáticos del contenido de las publicaciones (Tabla 3), podemos dividir la producción en dos ejes fundamentales: Fundamentación teórica y divulgación del Mobile Learning o Aprendizaje Móvil, con un $40 \%$ (76 documentos) y Experiencias y estudios de caso de implementación de esta modalidad educativa, con el $60 \%$ restante (114 documentos).

TABLA 3 - EJES TEMÁTICOS DEL CONTENIDO DE LA PRODUCCIÓN CIENTÍTICA DEL MOBILE LEARNING EN ESPAÑA (2009-2013)

\begin{tabular}{cccccccc}
\hline $\begin{array}{c}\text { Ejes temáticos de la producción científica sobre } \\
\text { Mobile Learning en España por años }\end{array}$ & $\mathbf{2 0 0 9}$ & $\mathbf{2 0 1 0}$ & $\mathbf{2 0 1 1}$ & $\mathbf{2 0 1 2}$ & $\mathbf{2 0 1 3}$ & $\mathbf{T}$ \\
\hline \multirow{2}{*}{ Fundamentación teórica y divulgación } & 2 & 13 & 11 & 25 & 24 & 76 \\
& & & & & & \\
& $\begin{array}{l}\text { Teléfono móvil/ } \\
\text { Smartphones } \\
\text { Tabletas }\end{array}$ & 2 & 4 & 3 & 3 & 3 & 14 \\
& digitales/iPad & 0 & 0 & 2 & 11 & 11 & 24 \\
& $\begin{array}{l}\text { SMS } \\
\text { Podcast }\end{array}$ & 0 & 1 & 0 & 0 & 0 & 1 \\
Experiencias y estudios de caso de & 1 & 2 & 6 & 1 & 4 & 14 \\
implementación & $\begin{array}{l}\text { Redes sociales } \\
\text { Realidad }\end{array}$ & 1 & 1 & 0 & 3 & 2 & 7 \\
& $\begin{array}{l}\text { Aumentada } \\
\text { Códigos QR }\end{array}$ & 0 & 1 & 3 & 7 & 7 & 18 \\
Apps & 0 & 0 & 1 & 5 & 3 & 9 \\
\hline TOTALES ANUALES & & 6 & 23 & 26 & 61 & 74 & 190 \\
\hline
\end{tabular}


En la siguiente tabla se recogen la relación de documentos del Anexo I que corresponden a cada núcleo temático (Tabla 4):

TABLA 4 - RELACIÓN DEL DOCUMENTOS POR TEMÁTICAS DEL ANEXO I

\begin{tabular}{|c|c|c|}
\hline \multicolumn{3}{|c|}{ Relación del documentos por temáticas del Anexo I } \\
\hline \multicolumn{2}{|c|}{ Fundamentación teórica y divulgación } & $\begin{array}{l}3,4,5,6,7,8,9,13,19,20,21,22,26,30, \\
33,34,35,36,37,38,42,48,49,54,55,56, \\
58,61,63,64,65,66,70,71,72,77,79,84, \\
85,90,95,98,103,106,107,112,117,124, \\
125,130,133,136,139,145,146,147,151, \\
153,157,159,160,161,162,164,168,169, \\
170,172,173,175,177,180,181,184,185, \\
186,190 .\end{array}$ \\
\hline \multirow{8}{*}{$\begin{array}{l}\text { Experiencias } \\
\text { y estudios } \\
\text { de caso de } \\
\text { implementación }\end{array}$} & Teléfono móvil/Smartphones & $\begin{array}{l}1,11,28,31,32,62,76,81,97,105,121,122, \\
126,137 .\end{array}$ \\
\hline & Tabletas digitales/iPad & $\begin{array}{l}15,27,29,39,52,59,68,69,73,74,88,89, \\
101,102,108,111,114,118,141,152,165, \\
166,167,176 .\end{array}$ \\
\hline & SMS & 31 \\
\hline & Podcast & $\begin{array}{l}14,40,57,67,75,82,83,86,94,104,110 \\
144,178,179 .\end{array}$ \\
\hline & Redes sociales & $44,78,123,131,138,140,142$ \\
\hline & Realidad Aumentada & $\begin{array}{l}16,23,24,43,47,50,93,109,113,119,120 \\
129,149,150,154,155,158,174\end{array}$ \\
\hline & Códigos QR & $17,41,53,91,92,96,171,187,188$. \\
\hline & Apps & $\begin{array}{l}2,10,12,18,25,45,46,51,60,80,87,99, \\
100,115,116,127,128,134,135,143,148, \\
156,162,182,183,189 .\end{array}$ \\
\hline
\end{tabular}

En cuanto a los autores, se han recogido los más recurrentes (con al menos dos citas) en la producción científica del Mobile Learning en España en el período estudiado y su campo de estudio, siendo los resultados los siguientes (Tabla 5):

TABLA 5 - AUTORES DE MOBILE LEARNING EN ESPAÑA

\begin{tabular}{lll}
\hline Autor & Documentos & Núcleos temáticos \\
\hline BRAZUELO, F. & $1,8,9,31,32,121,122,123$, & Fundamentación, Teléfono Móvil, Apps \\
& $124,125,126,127,128$ & \\
CLARES, J. & 138,140 & Redes sociales, Twitter \\
GÓMEZ, M. & $149,150,151,152,153$, & Realidad Aumentada \\
& 154,155 & \\
GROS, B. & 18,50 & Apps \\
LARA, T. & $20,21,56$ & Fundamentación, Software libre, Android \\
MIRALPEIX, A. & 68,69 & Tabletas, iPad \\
\hline
\end{tabular}


(conclusión)

TABLA 5 - AUTORES DE MOBILE LEARNING EN ESPAÑA

\begin{tabular}{lll}
\hline OLMEDO, K. & $49,168,169$ & Fundamentación \\
PARDO, H. & 78,79 & Redes sociales, Apps \\
RAMOS, A. & 85,86 & Podcast \\
RIVES, M. & 88,89 & Tabletas digitales \\
ROMÁN, P. & $91,92,175$ & Códigos QR \\
SÁNCHEZ, M. & $95,96,97$ & Fundamentación, Códigos QR \\
SANTIAGO, R. & $25,74,99,100$ & Tabletas digitales, iPad, Apps \\
SEVILLANO, M. & 26,103 & Apps \\
ZAMORA, J. L. & $187,188,189$ & Códigos QR \\
\hline
\end{tabular}

\section{Discusión y conclusiones}

El estado del Mobile Learning en España, según los datos de producción científica nacional entre inicios del 2009 y finales del 2013, es de creciente interés por la integración de las tecnologías móviles y sus funcionalidades en diversos ámbitos educativos y de formación, siendo especialmente significativo el aumento de la producción en los años 2012 y 2013.

Las áreas de investigación y experimentación del Mobile Learning han corrido paralelas a la evolución tecnológica de los propios dispositivos móviles. A medida que se incorporaban funcionalidades, se incrementó el interés de los investigadores en explorar sus potenciales usos educativos. De este modo, encontramos los teléfonos móviles, como medio tecnológico, y los SMS, los Podcast y las Redes Sociales como las funcionalidades con más investigaciones en la primera parte del período estudiado. Sin embargo, en una segunda etapa, destacan las tabletas digitales, las Apps o aplicaciones móviles además de la Realidad Aumentada y los Códigos QR.

Teniendo en cuenta el impacto social y educativo de las tecnologías móviles, por un lado, y la comparativa de la producción científica nacional sobre Mobile Learning con la total publicada en España en este período en las fuentes consultadas, puede considerarse esta como escasa.

Por tanto, ha habido un empuje del Mobile Learning en España en los dos últimos años, pero su estado es aún incipiente. Se hace necesario, entre otras actuaciones: aceptar las tecnologías móviles en los contextos escolares y de formación, regulando su uso; impulsar políticas educativas de implantación de estas tecnologías; difundir ejemplos de uso de Mobile Learning; fomentar la creación de contenidos educativos móviles; y lo más importante, preparar al profesorado como elemento clave para la integración real de las tecnologías móviles con fines educativos. 


\section{REFERENCIAS}

BRAZUELO, F.; GALLEGO, D. J. Mobile Learning: los dispositivos móviles como recurso educativo. Sevilla: MAD Eduforma, 2011.

DAY, R. Cómo escribir y publicar trabajos científicos. Washington: Organización Panamericana de Salud, 2005.

GEORGIEV, T.; GEORGIEVA, E.; TRAJOVSKI, G. Transitioning from e-Learning to m-Learning. Software Engineering, n. 57, p. 349-353, 2006.

GUIRAO-GORIS, J. A; OLMEDO, A; FERRER, E. El artículo de revisión. Revista Iberoamericana de Enfermería Comunitaria, v. 1, n. 6, 2008. Disponible en: <http://revista.enfermeriacomunitaria.org/articuloCompleto.php?ID=7>. Acceso en: 27 dic. 2013.

KEEGAN, D. Mobile Learning: the next generation of learning. 2005. Disponible en: $<$ http://learning.ericsson.net/mlearning2/resources.shtml>. Acceso en: 22 mar. 2012.

O’MALLEY, C.; VAVOULA, G.; GLEW, J.; TAYLOR, J.; SHARPLES, M. Guidelines for learning/teaching/tutoring in a mobile environment. 2005. Disponible en: $<$ http:// www.mobilearn.org/download/results/guidelines.pdf>. Acceso en: 22 mar. 2012.

PACHLER, N.; BACHMAIR, B.; COOK, J. Mobile Learning: Structures, Agency, Practices. London: Springer, 2010.

WOODILL, G. The Mobile Learning Edge. New York: McGrawHill, 2010.

UNESCO. Policy Guidelines for Mobile Learning. 2013. Disponible en: < http://unesdoc. unesco.org/images/0021/002196/219641E.pdf>. Acceso en: 10 dic. 2013.

\section{ANEXO I - Publicaciones científicas sobre Mobile Learning en España (2009-2013)}

\section{Tesis Doctorales}

1. BRAZUELO, F. El teléfono móvil: actitudes, usos y posibilidades educativas. Tesis (Doctorado) - Facultad de Educación, Universidad Nacional de Educación a Distancia, Madrid, 2013.

2. FERNÁNDEZ, A. Sistemas de Mobile Learning para alumnado con Necesidades Especiales. Tesis (Doctorado) - Facultad de Ingeniería Informática, Universidad de Granada, Granada, 2013. 


\section{Libros e Ebooks}

3. BRAZUELO, F.; GALLEGO, D. J. Mobile Learning: los dispositivos móviles como recurso educativo. Sevilla: MAD Eduforma, 2011.

4. CASTAÑO, G.; CABERO, J. (Coords.). Enseñar y aprender en entornos m-learning. Madrid: Síntesis, 2013.

5. POVEDANO, A.; ORO, P. Mlearning: la formación en tu móvil. A Coruña: NetBiblo, 2009.

6. SCOPEO. M-learning en España, Portugal y América Latina, Noviembre de 2011. Disponible en: <http://scopeo.usal.es/wp-content/uploads/2013/04/scopeom003.pdf>. Acceso en: 3 mar. 2012.

7. RON, R.; ÁLVAREZ, A.; NUÑEZ, P. (Coords.). Smartphones y Tablets: ¿Enseñan o distraen? Madrid: ESIC, 2013.

\section{Capítulos de Libros}

8. BRAZUELO, F. Nuevos recursos móviles. En: GALLEGO, D.; ALONSO, C. M.; CACHEIRO, M. L. (Coords.). Educación, Sociedad y Tecnología. Madrid: Editorial Ramón Areces, 2011. Capítulo 13. p. 337-366.

9. BRAZUELO, F. Mobile Learning o Aprendizaje Móvil. En: CACHEIRO, M. L (Coord). Educación y Tecnología: Estrategias y Contenidos Digitales. Madrid: Editorial UNED, 2013.

10. CADENAS, C. Cinco puntos imprescindibles para el éxito de una "app" infantil o juvenil. En: ÁLVAREZ, A; NUÑEZ, P.; RODRIGO, R. (Coords.). Smartphones y tablets: ¿enseñan o distraen? Madrid: ESIC, 2013. p. 45-48.

11. CASTELLAR, C.; PRADAS, F.; COLL, I. Utilización de las nuevas tecnologías en la asignatura Actividades deportivas en el medio acuático en la Diplomatura de Maestro Especialista en Educación Física: el uso del teléfono móvil. En: ALEJANDRE, J. L. (Coord.). Buenas prácticas en la docencia universitaria con apoyo de TIC. Zaragoza: Universidad de Zaragoza, 2012. p. 149-156.

12. CHUECA, N. “Apps” y plataformas: un espacio infinito para el juego en Imaginarium. En: ÁLVAREZ, A; NUÑEZ, P.; RODRIGO, R. (Coords.). Smartphones y tablets: ¿enseñan o distraen? Madrid: ESIC, 2013. p. 37-44.

13. CONTRERAS, R. Aprendizaje móvil y cognitivo: un estudio de caso. En: CONTRERAS, R.; GARCÍA, I. (Coords.). m-Todos: tendencias y oportunidades de la movilidad digital. Barcelona: Universidad de Vic, 2012. p. 201-209.

14. DARIAS, A.; SORAYA, M.; VIDAL, R. Aprendizaje móvil, ubicuo y autónomo de lenguas extranjeras en la ULPGC. En: LLAVERO, E. (Coord.). Cuadernos de Innova- 
ción Educativa. Las Palmas: Servicio de Publicaciones y Difusión Científica ULPGC, 2013. p. 11-35.

15. ECHANOVE, M. De móviles y "tablets", niños y jóvenes, colegios y tiempo de ocio. En: ÁlVAREZ, A; NUÑEZ, P.; RODRIGO, R. (Coords.). Smartphones y tablets: ¿enseñan o distraen? Madrid: ESIC, 2013. p. 67-76.

16. ESTEBANELL, M.; FERRÚS, J.; CORNELLÁ, P.; CODINA, D. Realidad Aumentada y Códigos QR en educación. En: HERNÁNDEZ, J.; PENNESI, D.; VÁZQUEZ, A. (Coords.). Tendencias emergentes en educación con TIC. Barcelona: Espiral, 2012. p. $135-157$.

17. GAMBOA, J. L. El uso de códigos QR en la enseñanza. En: HERNÁNDEZ, J.; PENNESI, D.; VÁZQUEZ, A. (Coords.). Tendencias emergentes en educación con TIC. Barcelona: Espiral, 2012. p. 197-211.

18. GROS, B. Aplicaciones móviles para educación. En: CABERO, J.; AGUADED, J. (Coords.). Tecnologías y medios para la educación en la e-sociedad. Madrid: Alianza Editorial, 2013. p. 71-90.

19. ITEM FORMACIÓN UOC. Creación de una plataforma y metodología colaborativa para el M-Learning. En: ETXEBARRÍA, L. (Coord.). Nuevas tendencias de e-learning y actividades didácticas innovadoras. Madrid: Ediciones CEF, Universidad a Distancia de Madrid, 2010. p. 117-122.

20. LARA, T. Mobile Learning EOI: abriendo el aula. En: HERNÁNDEZ, J.; PENNESI, M.; SOBRINO, D.; VÁZQUEZ, A. Experiencias educativas en las aulas del siglo XXI. Barcelona: Espiral, 2011. p. 252-254.

21. LARA, T. MLearning. Cuando el Caballo de Troya entró en el aula. En: HERNÁNDEZ, J.; PENNESI, M.; VÁZQUEZ, A. (Coords.). Tendencias emergentes en educación con TIC. Barcelona: Espiral, 2012.

22. MONTEAGUDO, J. Dispositivos móviles en el aula: el aprendizaje en nuestras manos. En: HERNÁNDEZ, J.; PENNESI, D.; VÁZQUEZ, A. (Coords.). Tendencias emergentes en educación con TIC. Barcelona: Espiral, 2012. p. 245.263.

23. MORAL, E.; VILLALUSTRE, L. Realidad aumentada: experimentando en el aula en 3D. En: ÁLVAREZ, A; NUÑEZ, P.; RODRIGO, R. (Coords.). Smartphones y tablets: ¿enseñan o distraen? Madrid: ESIC, 2013. p. 109-126; 109-126.

24. REINOSO, R. Posibilidades de la Realidad Aumentada en educación. En: HERNÁNDEZ, J.; PENNESI, D.; VÁZQUEZ, A. (Coords.). Tendencias emergentes en educación con TIC. Barcelona: Espiral, 2012. p. 175-197.

25. SANTIAGO, R. Tecnología móvil e innovación en el aula: nuevos retos y realidad educativas. En: GOIG, R. (Coord.). Formación del profesorado en la sociedad digital. Investigación, innovación y recursos didácticos. Madrid: Editorial UNED, 2013. 
26. SEVILLANO, M. L. Enseñanza y Aprendizaje con dispositivos móviles. En: CABERO, J.; AGUADED, J. (Coords.). Tecnologías y medios para la educación en la e-sociedad. Madrid: Alianza Editorial, 2013. p. 159-184.

27. SOLÍS, J. La rápida entrada de las "tablets" en el mercado infantil: razones y reflexiones. En: ÁLVAREZ, A; NUÑEZ, P.; RODRIGO, R. (Coords.). Smartphones y tablets: ¿enseñan o distraen? Madrid: ESIC, 2013. p. 77-94.

28. VICENT, N.; ASENSIO, M.; IBÁNEZ, A. Evaluación de las unidades educativas "Zarautz en tus manos" y "Expedición Menosca". Aprendizaje informal a través del patrimonio mediante el uso de tecnología móvil. En: MILLARES, P.; MOLINA, S.; SANTISTEBAN, A. (Coords.). La evaluación en el proceso de enseñanza y aprendizaje de las Ciencias Sociales. Murcia: Asociación Universitaria de Profesorado de Didáctica de las Ciencias Sociales, 2011. p. 305-312.

\section{Artículos de Revistas Científicas}

29. APARICIO, R. Las tabletas y el TEA: una herramienta para la inclusión en la escuela ordinaria. 2013. Disponible en: <http://aula.grao.com/revistas/aula/223_224-el-gusto-por-aprender/las-tabletas-y-el-tea-una-herramienta-para-la-inclusion-en-la-escuela-ordinaria>. Acceso en: 19 dic. 2013.

30. ARJONA, J.; GÁMIZ, V. Revisión de opciones para el uso de la plataforma Moodle en dispositivos Móviles. Revista de Educación a Distancia, n. 37, 2013. Disponible en: $<$ http://www.um.es/ead/red/37/arjona.pdf>. Acceso en: 19 dic. 2013.

31. BRAZUELO, F.; GALLEGO, D. Sistema de Gestión Tutorial vía SMS e Internet. Pixel Bit: Revista de Medios y Educación, n. 34, p. 49-67, 2010.

32. BRAZUELO, F.; CACHEIRO, M. L. Diseño de páginas web educativas para teléfonos móviles. Edutec: Revista Electrónica de Tecnología Educativa, n. 32, 2010. Disponible en: <http://edutec.rediris.es/Revelec $2 /$ revelec32/dise\%C3\%B1o_web_educativas_telefonos_moviles.html $>$. Acceso en: 17 dic. 2013.

33. CAMACHO, M. Mobile learning: aproximación conceptual y prácticas colaborativas emergentes. Universitas tarraconensis: Revista de Ciències de L'educació, n. 2, p. 43-50, 2011.

34. CAMPO, E. M-Learning y aprendizaje informal en la educación superior mediante dispositivos móviles. Historia y Comunicación Social, n. 18, p. 213-242, 2013.

35. CAÑELLAS, A. Introducción al m-learning. Comunicación y Pedagogía: Nuevas Tecnologías y Recursos Didácticos, n. 259-260, p. 38-40, 2012.

36. CASALS, R. El móvil y la capacidad de reflexión y profundización. 2013. Disponible en: <http://aula.grao.com/revistas/aula/223_224-el-gusto-por-aprender/el-movil-y-la-capacidad-de-reflexion-y-profundizacion>. Acceso en: 19 dic. 2013. 
37. CASAÑA, M. J.; BARCELÓ, M. Cómo definir proyectos de m-learning más sostenibles. Teoría de la Educación: Educación y Cultura en la Sociedad de la Información, v. 14, n. 2, p. 271-291, 2013.

38. CATALDI, Z.; LAGE, F. Entornos personalizados de aprendizaje (EPA) para dispositivos móviles: situaciones de aprendizaje y evaluación. EDMETIC, v. 2, n. 1, p. 117-148, 2013.

39. CASTELLANO, R.; SÁNCHEZ, R. Laptop, andamiaje para la Educación Especial. Guía práctica. Computadoras móviles en el currículo. Revista Didáctica, Innovación y Multimedia, n. 22, 2012. Disponible en: <http://www.pangea.org/dim/revista22>. Acceso en: 18 dic. 2013.

40. CHACÓN, C.; PÉREZ, C. El podcast como innovación en la enseñanza del inglés como lengua extranjera. Pixel Bit: Revista de Medios y Educación, n. 39, p. 41-52, 2011.

41. DOMÉNECH, R. Códigos QR como propuesta de trabajo desde el área de música. Eufonia, n. 52, p. 25-34, 2011.

42. DOMÍNGUEZ, S.; AGUDO, E.; SÁNCHEZ, H. Análisis comparativo de interfaces de usuario para video interactivo educativo en dispositivos móviles. IE Comunicaciones: Revista Iberoamericana de Informática Educativa, n. 17, p. 3-12, 2013.

43. FOMBONA, J.; PASCUAL, M.; MADEIRA, A. Realidad aumentada, una evolución de las aplicaciones de los dispositivos móviles. Pixel Bit: Revista de Medios y Educación, n. 41, p. 197-210, 2012.

44. FUENTES, L. Creación de un patrón de eLearning a partir de la consideración de aspectos relacionados con el diseño de objetos de aprendizaje para un caso práctico concreto de uso del móvil para dar soporte a Lifelong Learners, desde la perspectiva del diseño instruccional. Revista de Educación a Distancia, n. 31, 2012. Disponible en: $<$ http://www.um.es/ead/red/31/cesar.pdf>. Acceso en: 19 dic. 2013.

45. GARCÍA, A.; REY, R. Las apps en el aula del siglo XXI. Comunicación y Pedagogía: Nuevas Tecnologías y Recursos Didácticos, n. 259-260, p. 32-37, 2012.

46. GARCÍA, E.; TORRENTERAS, J. "Las intervenciones de enfermería” como patrón pedagógico de e-learning, wiki y aplicación móvil. Revista de Educación a Distancia, n. 39, 2013. Disponible en: <http://www.um.es/ead/red/39/ElenaGarcia.pdf>. Acceso en: 19 dic. 2013.

47. GONZÁLEZ, A.; CHÁVEZ, G. La realidad virtual inmersiva en ambientes inteligentes de aprendizaje: un caso en la educación superior. Icono14, v. 9, n. 2, p. 122-137, 2011.

48. GÓMEZ HERNÁNDEZ, P.; MONGE LÓPEZ, C. Potencialidades del teléfono móvil como recurso innovador en el aula: una revisión teórica. Revista Didáctica, Innovación y Multimedia, n. 26, 2013. Disponible en: <http://www.pangea.org/dim/revista26>. Acceso en: 18 dic. 2013. 
49. GRANÉ, M.; CRESCENDI, L.; OLMEDO, K. Cambios en el uso y la concepción de las TIC, implementando el Mobile Learning. Revista de Educación a Distancia, n. 37 , 2013. Disponible en: <http://www.um.es/ead/red/37/grane.pdf>. Acceso en: 19 dic. 2013.

50. GROS, B.; FORÉS, A. El uso de la geolocalización en educación secundaria para la mejora del aprendizaje situado: Análisis de dos estudios de caso. RELATEC: Revista Latinoamericana de Tecnología, n. 12, p. 41-53, 2013.

51. HERNÁNDEZ, J. Móviles y Apps. Comunicación y Pedagogía: Nuevas Tecnologías y Recursos Didácticos, n. 259-260, p. 41-46, 2012.

52. IBÁÑEZ, A.; VICENT, N.; ASENSIO, A. Aprendizaje informal, patrimonio y dispositivos móviles: evaluación de una experiencia en educación secundaria. Didáctica de las Ciencias Experimentales y Sociales, n. 26, p. 3-18, 2012.

53. IZQUIERDO, A. Códigos QR flexibles: un proyecto con dispositivos móviles para el trabajo de calentamiento en educación física. EmásF: Revista Digital de Educación Física, n. 23, p. 53-71, 2013.

54. JOHNSON, L.; ADAMS BECKER, S.; CUMMINS, M., ESTRADA, V.; FREEMAN, A.; LUDGATE, H. Aprendizaje Mediante Dispositivos Móviles. 2013. Disponible en: $<$ http://www.eduteka.org/dispositivosmoviles.php>. Acceso en: 19 dic. 2013.

55. KANTEL, E.; TOVAR, G.; SERRANO, A. Diseño de un Entorno Colaborativo Móvil para Apoyo al Aprendizaje a través de Dispositivos Móviles de Tercera Generación. IEEE-Rita, v. 5, n. 4, p. 146-152, 2010.

56. LARA, T. Mobile learning EOI: Android, una apuesta por el conocimiento abierto. Telos: Cuadernos de Comunicación e Innovación, n. 83, p. 1-4, 2010.

57. LAASER, W.; JASKILIOFF, S.; RODRÍGUEZ, L. Postcasting: ¿Un nuevo medio para la Educación a Distancia? Revista de Educación a Distancia, n. 23, 2010. Disponible en: <http://www.um.es/ead/red/23/laaser.pdf>. Acceso en: 19 dic. 2013.

58. LAMASTER, J.; STAGER, G. ¿Deben usar los estudiantes en el aula sus propios dispositivos móviles (BYOD)? 2012. Disponible en: <http://www.eduteka.org/modulos/8/243/2130/1>. Acceso en: 19 dic. 2013.

59. LÓPEZ, F.; RUÍZ, M. Elaboración de material docente para iPad con iBooks Author. Epsilon: Revista de la Sociedad Andaluza de Educación Matemática, n. 83, 2013. Disponible en: <http://thales.cica.es/epsilon/?q=content/elaboraci\%C3\%B3n-de-material-docente-para-ipad-con ibooks-author>. Acceso en: 19 dic. 2013.

60. LOZANO, I.; SAFONT, LL.; LUQUE, A. Motivar y aprender con el móvil creando una aplicación para Android, mediante una metodología lúdica, constructivista y social. Revista de Educación a Distancia, n. 36, 2013. Disponible en: <http://www.um.es/ead/ red/36/lozano.pdf>. Acceso en: 19 dic. 2013.

61. MADRID, D.; MAYORGA, M.; NUÑEZ, F. Aplicación del M-learning en el aula de primaria: experiencia práctica y propuesta de formación para docentes. Edutec: Revista 
Electrónica de Tecnología Educativa, n. 45, 2013. Disponible en: < http://edutec.rediris.es/ Revelec2/Revelec45/aplicacion_mlearning_primaria_experiencia_formacion_docentes. html>. Acceso en: 17 dic. 2013.

62. MAGGIOLINI, L. Estrategias de Motivación en una Era Digital: Teléfonos móviles y Facebook en el aula. Digital Education Review, n. 24, 2013. Disponible en: <http:// greav.ub.edu/der/index.php/der/article/viewArticle/239>. Acceso en: 18 dic. 2013.

63. MALEK, J.; LAROUSSI, M.; DERYCKE, A.; GHEZALA, H. Una metodología de diseño para el aprendizaje ubicuo. Novática: Revista de la Asociación de Técnicos de Informática, n. 210, p. 46-50, 2011.

64. MARTÍNEZ, M.; HILERA, J. Nueva aplicación m-learning diseñada para la autoevaluación. Pulso. Revista de Educación, n. 33, 2010. Disponible en: <http://dspace.uah. es/dspace/handle/10017/7212>. Acceso en: 19 dic. 2013.

65. MARQUEZ, J.; LAUTERO, J. Implementación del servicio de mobile-learning para la universidad Antonio Nariño. Revista Didáctica, Innovación y Multimedia, n. 24, 2012. Disponible en: <http://www.pangea.org/dim/revista> Acceso en: 18 dic. 2013.

66. MEDINA, S. U-Learning. El futuro está aquí. RUSC. Universities and Knowledge Society Journal, v. 7, n. 2, p. 1-3, 2010.

67. MELGAREJO, I.; RODRÍGUEZ, M. La radio como recurso didáctico en el aula de infantil y primaria: los podcast y su naturaleza educativa. Tendencias Pedagógicas, n. 21, p. 29-46, 2013.

68. MIRALPEIX, A. Recursos del iPad i del iPhone aplicados a la educación universitaria de la música. Aloma. Revista de Psicologia, Ciències de l'Educació i de l'Esport, v. 1, n. 31, p. 33-42, 2013.

69. MIRALPEIX, A. iMúsica: educación musical con el iPad y el iPhone. Eufonía, n. 56, p. 27-35, 2012.

70. MOLINA, A.; CHIRINO, V. Mejores Prácticas de Aprendizaje Móvil para el Desarrollo de Competencias en la Educación Superior. IEEE-Rita, v. 5, n. 4, p. 175-183, 2010.

71. MOLINA, J.; ROMERO, D. Ambiente de Aprendizaje Móvil Basado en Micro-Aprendizaje, v. 5, n. 4, p. 159-166, 2010.

72. MUÑOZ, C. Dispositivos móviles en la educación médica. Teoría de la Educación: Educación y Cultura en la Sociedad de la Información, v. 11, n. 2, p. 28-45, 2010.

73. NAKAKO, T.; GARRET, P.; MIJA, A.; VELASCO, A.; BEGAZO, J.; ROSALES, A. Uso de tablets en la educación superior: una experiencia con iPads. Digital Education Review, n. 24, 2013. Disponible en: <http:/greav.ub.edu/der/index.php/der/article/ viewArticle/241>. Acceso en: 18 dic. 2013.

74. NAVARINAS, F.; SANTIAGO, R.; TOURÓN, J. Valoraciones del profesorado del área de Fresno (California Central) sobre la influencia de la tecnología móvil en el 
aprendizaje de sus estudiantes. RELIEVE, n. 19, 2013. Disponible en: < http://www.uv.es/ RELIEVE/v19n2/RELIEVEv19n2_4.htm>. Acceso en: 19 dic. 2013.

75. NUEZ, C. Podcast, música y educación. Eufonía, n. 52, p. 7-15, 2011.

76. ORGANISTA, J.; SERRANO, A. Implementación de una actividad educativa en modo colaborativo con apoyo de smarthpones: una experiencia universitaria. Edutec: Revista Electrónica de Tecnología Educativa, n. 36, 2011. Disponible en: $<$ http://edutec. rediris.es/Revelec2/Revelec36/pdf/Edutec-e_n36_Organista_Serrano.pdf $>$. Acceso en: 17 dic. 2013.

77. ORTIGOSA, A.; BRAVO, J.; CARRO, R.; MARTÍN, E. Entornos de aprendizaje móviles adaptativos y evaluación: CoMoLE y GeSES. RIED: Revista Iberoamericana de Educación a Distancia, v. 13, n. 2, p. 167-207, 2010.

78. PARDO, H. Campus móvil. La primera red social universitaria vía dispositivos móviles de iberoamérica. Un estudio de caso. RIED: Revista Iberoamericana de Educación a Distancia, v. 12, n. 2, p. 21-32, 2009.

79. PARDO, H.; BALESTRINI, M. Prototipos de Mobile Open Education: Una breve selección de Casos. IEEE-Rita, v. 5, n. 4, p. 125-131, 2010.

80. PEGALAJAR, M.; COLMENERO, M. PICAA: aplicación móvil de aprendizaje para la inclusión educativa del alumnado con discapacidad.Etic@net, n. 14,p.94-106, 2013.

81. PISANTY, A.; ENRÍQUEZ, L.; CHAOS, L.; GARCÍA, M. "M-Learning en Ciencia": introducción de aprendizaje móvil en física. RIED: Revista Iberoamericana de Educación a Distancia, v. 13, n. 1, p. 129-155, 2010.

82. PIÑEIRO, M.; COSTA, C. Potencialidades del Podcast como herramienta educativa para la enseñanza universitaria.Etic@net, n. 1, p. 124-136, 2011.

83. QUINTANAL, F. El podcast como herramienta de enseñanza en física y química de bachillerato. Estudios Sobre el Mensaje Periodístico, n. 18, p. 729-738, 2012.

84. RAMÍREZ, M. Recursos tecnológicos para el aprendizaje móvil (mlearning) y su relación con los ambientes de educación a distancia: implementaciones e investigaciones. RIED: Revista Iberoamericana de Educación a Distancia, v. 12, n. 2, p. 57-82, 2009.

85. RAMOS, A.; HERRERA, J.; RAMÍREZ, M. Desarrollo de habilidades cognitivas con aprendizaje móvil: un estudio de casos. Comunicar: Revista Científica Iberoamericana de Comunicación y Educación, n. 34, p. 201-209, 2010.

86. RAMOS, A.; CAURCEL, M. Los podcast como herramienta de enseñanza-aprendizaje en la universidad. Profesorado: Revista de Curriculum y Formación del Profesorado, n. 1, p. 151-162, 2011.

87. RIAL, T.; VILLANUEVA, C. Las aplicaciones para terminales móviles como herramienta didáctica en el desarrollo de contenidos rítmicos y expresivos. EmásF: Revista Digital de Educación Física, n. 23, p. 7-15, 2013. 
88. RIVES, M. Las tabletas en la educación del siglo XXI. Eufonía, n. 56, p. 7-19, 2012. 89. RIVES, M. Aprender con dispositivos móviles: las tabletas. 2013. Disponible en: $<$ http://aula.grao.com/revistas/aula/218-profesorado-2013/aprender-con-dispositivos-moviles-las-tabletas>. Acceso en: 19 dic. 2013.

90. ROBLES, H.; FONTALVO, H., GUERRA, D. Desarrollo de habilidades escriturales apoyado con tecnología móvil. Teoría de la Educación: Educación y Cultura en la Sociedad de la Información, v. 13, n. 3, p. 380-402, 2013.

91. ROMÁN, P. Design, Creation and Implementation of QR's Code Virtual Observatory. @tic. Revista de Innovación Educativa, n. 9, 2012. Disponible en: $<$ http://ojs.uv.es/index. php/attic/article/view/1947>. Acceso en: 18 dic. 2013.

92. ROMÁN, P.; MARTÍN, A. La formación de docentes en estrategias innovadoras de enseñanza y aprendizaje: los códigos de respuesta rápida o códigos QR. Revista Didáctica, Innovación y Multimedia, n. 26, 2013. Disponible en: <http://www.pangea. org/dim/revista26>. Acceso en: 18 dic. 2013.

93. RUIZ, D. Realidad Aumentada, educación y museos. Icono14, v. 9, n. 2, p. 212-226, 2011.

94. RUTH, S.: EGUIA, J. L. Contenidos de aprendizaje para estudiantes de diseño en podcast. Cuadernos de Documentación Multimedia, n. 20, p. 139-148, 2009.

95. SÁNCHEZ, M. Uso del dispositivo móvil como recurso digital. Revista Didáctica, Innovación y Multimedia, n. 22, 2012. Disponible en: < http://www.pangea.org/dim/ revista22>. Acceso en: 18 dic. 2013.

96. SÁNCHEZ, M. Los códigos QR invaden los foros de discusión. Revista Didáctica, Innovación y Multimedia, n. 25, 2013. Disponible en: < http://www.pangea.org/dim/ revista25>. Acceso en: 18 dic. 2013.

97. SÁNCHEZ, M. El uso del celular para desarrollar el pensamiento crítico, reflexivo y analítico.Etic@net, n. 11,n. 11,p.196-212,2011.

98. SANDOVAL, E.; GARCÍA, R.; RAMÍREZ, M. Competencias tecnológicas y de contenido necesarias para capacitar en la producción de recursos de aprendizaje móvil. Edutec: Revista Electrónica de Tecnología Educativa, n. 39, 2012. Disponible en: $<$ http:// edutec.rediris.es/Revelec2/Revelec39/pdf/Edutec-e_39_Sandoval_Garcia_Ramirez.pdf>. Acceso en: 17 dic. 2013.

99. SANTIAGO, R.; DÍEZ, A.; NAVARINAS, F. Diseño de una app educativa para dispositivos móviles: el proyecto del Pájaro Caracol. Comunicación y Pedagogía: Nuevas Tecnologías y Recursos Didácticos, n. 259-260, p. 47-51, 2012.

100. SANTIAGO, R.; DÍEZ, A.; NAVARINAS, F. Catalogación y análisis de la calidad de apps para dispositivos móviles: el Proyecto Eduapps. Comunicación y Pedagogía: Nuevas Tecnologías y Recursos Didácticos, n. 259-260, p. 52-55, 2012. 
101. SAORÍN, J. L.; TORRE, J.; MARTÍN, N.; CARBONELL, C.; CONTERO, M. Tabletas digitales para la docencia del dibujo, diseño y artes plásticas. Teoría de la Educación: Educación y Cultura en la Sociedad de la Información, v. 12, n. 2, p. 259-279, 2011.

102. SÁNCHEZ, J. L. La magia de aprender con los Dedos. Eufonía, n. 56, p. 36-42, 2012. 103. SEVILLANO, M. L.; VÁZQUEZ, E. La universidad ante el reto del aprendizaje ubicuo con dispositivos móviles. Edetania, n. 44, p. 33-46, 2013.

104. SOLANO, I.; SÁNCHEZ, M. Aprendiendo en cualquier lugar: el podcast educativo. Pixel Bit: Revista de Medios y Educación, n. 36, p. 125-139, 2010.

105. SOTELO, J. Del "e-learning" al "m-learning": una academia en cada "iPhone". Telos: Cuadernos de comunicación e innovación, n. 81, p. 122-128, 2009.

106. SUÁREZ, R.; CRESCENZI, L.; GRANÉ, M. Análisis del entorno colaborativo creado para una experiencia de mobile learning. Teoría de la Educación: Educación y Cultura en la Sociedad de la Información, v. 14, n. 1, p. 101-122, 2013.

107. TABUENCA, B.; VERPOORTEN, S.; TERNIER, S; WESTERA, W.; SPECHT, M. Fomento de la práctica reflexiva sobre el aprendizaje mediante el uso de tecnologías móviles. Revista de Educación a Distancia, n. 37, 2013. Disponible en: <http://www. um.es/ead/red/37/tabuenca2.pdf>. Acceso en: 19 dic. 2013.

108. TESTA, A.; MORODER, K. ¿Pueden los iPads reemplazar los portátiles? 2013. Disponible en: <http://www.eduteka.org/sinoipad.php>. Acceso en: 19 dic. 2013.

109. TORRE, J.; MARTÍN, N.; SAORÍN, J. L.; CARBONELL, C.; CONTERO, M. Entorno de aprendizaje ubicuo con realidad aumentada y tabletas para estimular la comprensión del espacio tridimensional. Revista de Educación a Distancia, n. 37, 2013. Disponible en: <http://www.um.es/ead/red/37/DELATORREetAL.pdf>. Acceso en: 19 dic. 2013.

110. TRUJILLO, J. M. Comunicación, innovación, educación y gestión del conocimiento en torno al uso del podcast en la educación superior. RUSC. Universities and Knowledge Society Journal, v. 7, n. 2, p. 61-763, 2011.

111. WALTERS, E.; BAUM, M. ¿Revolucionará el iPad la educación? 2013. Disponible en: <http://www.eduteka.org/sino_ipad.php>. Acceso en: 19 dic. 2013.

112. ZAPATA, R. Calidad en entornos ubicuos de aprendizaje. Revista de Educación a Distancia, n. 31, 2012. Disponible en: <http://www.um.es/ead/red/31/zapata_ros.pdf > Acceso en: 19 dic. 2013.

\section{Artículos en Eventos Científicos}

113. ATRIO, S.; LOZANO, J. La Realidad Aumentada y su incorporación al aula. Tecnología y Metodología. En: GALLEGO, D.; ALONSO, C. (Coords.). CONGRESO INTERNACIONAL DE TECNOLOGÍAS PARA LA EDUCACIÓN Y EL CONOCIMIENTO, XVII., 2012, Madrid. UNED. Madrid: Anaya, 2012. ISBN: 978-84-695-3582-0. 
114. BALADRÓN, C.; AGUIAR, J. M.; CALAVIA, L.; CARRO, B.; SÁNCHEZ, A. Interactive learning application for mobile devices. International Conference on Education and New Learning Technologies (EDULEARN 2012). Disponible en: <http://library. iated.org/view/BALADRON2012INT.2012>. Acceso en: 22 dic. 2013.

115. BALSERA, F.; MARTÍN, M. Aplicaciones móviles para la educación musical. En: CACHEIRO, M. L.; GALlEGO, D.; ALONSO, C. (Coords.). CONGRESO INTERNACIONAL DE TECNOLOGÍAS PARA LA EDUCACIÓN Y EL CONOCIMIENTO. INTERESTRATIC, XVIII., 2013, Madrid. UNED. Madrid: Anaya, 2013. ISBN: 978-84695-7990-9.

116. BALLESTA, F.; ALCARAZ, S.; GARCÍA, C. Programación de aplicaciones para dispositivos móviles ("apps") como enriquecimiento curricular en sujetos de alta capacidad. En: CARDONA, M.; CHINER, E.; GINER, A. (Coords.). INTERNACIONAL MODELOS DE INVESTIGACIÓN EDUCATIVA DE LAASOCIACIÓN INTERUNIVERSITARIA DE INVESTIGACIÓN PEDAGÓGICA, II., 2013, Alicante. Alicante: Asociación Interuniversitaria de Investigación Pedagógica (AIDIPE) y Universidad de Alicante, 2013. p. 962-970.

117. BENAVIDES, O. M-Learning: Nuevos procesos y contextos educativos. Jornadas Internacional del Mobile Learning. En: NAVARIDAS, F.; SANTIAGO, R.; SOTA 1, J.; JIMÉNEZ, M. A.; GONZÁLEZ, L. (Coords.). Jornadas sobre tecnología móvil e innovación en el aula. Logroño, España, 2013.

118. BENAVIDES, W.; LAVANDERA, S. El iPad como metodología de aprendizaje: una experiencia en educación. En: CACHEIRO, M. L.; GALLEGO, D.; ALONSO, C. (Coords.). CONGRESO INTERNACIONAL DE TECNOLOGÍAS PARA LA EDUCACIÓN Y EL CONOCIMIENTO. InterESTRATIC, XVIII., 2013, Madrid. UNED. Madrid: Anaya, 2013. ISBN: 978-84-695-7990-9.

119. BONIN, J.; CABEZAS, S. Realidad Aumentada y Pizarras Digitales Interactivas. En: CACHEIRO, M. L.; GALLEGO, D.; ALONSO, C. (Coords.). CONGRESO INTERNACIONAL DE TECNOLOGÍAS PARA LA EDUCACIÓN Y EL CONOCIMIENTO. INTERESTRATIC, XVIII., 2013, Madrid. UNED. Madrid: Anaya, 2013. ISBN: 978-84695-7990-9.

120. BONO, A.; BONIFACIO, M. Pizarras y tabletas digitales en la Universidad: una experiencia docente en ingeniería. En: FIDALGO, A.; SEIN-ECHALUCE, M. L. (Coords.). CONGRESO INTERNACIONAL SOBRE APRENDIZAJE, INNOVACIÓN Y COMPETIVIDAD, II., 2013, Madrid. Madrid: Fundación General de la Universidad Politécnica de Madrid, 2013. p. 709-714.

121. BRAZUELO, F. Prohibido APAGAR el teléfono móvil. En: GALLEGO, D.; ALONSO, C. (Coords.). CONGRESO INTERNACIONAL DE TECNOLOGÍAS PARA LA 
EDUCACIÓN Y EL CONOCIMIENTO, XIV., 2009, Madrid. UNED. Madrid: Anaya, 2009. ISBN: 978-84-692-3571-3.

122. BRAZUELO, F. Mobile Web 2.0: Diseño y desarrollo de contenidos web educativos para teléfonos móviles. En: CONGRESO EDUTEC-E 2010, X., 2010. EPV, 2010.

123. BRAZUELO, F. Participación de las Redes Sociales en telefonía móvil en engadget. En: GALlEGO, D.; ALONSO, C. (Coords.). CONGRESO INTERNACIONAL DE TECNOLOGÍAS PARA LA EDUCACIÓN Y EL CONOCIMIENTO, XV., 2010, Madrid. UNED. Madrid: Anaya, 2010. ISBN: 978-84-693-2709-8.

124. BRAZUELO, F. Mobile Learning. En: GALlEGO, D.; ALONSO, C. (Coords.). CONGRESO INTERNACIONAL DE TECNOLOGÍAS PARA LA EDUCACIÓN Y EL CONOCIMIENTO, XVI., 2011, Madrid. UNED. Madrid: Anaya, 2011. ISBN: 978-972-8939-45-8.

125. BRAZUELO, F. Experiencias y proyectos de aplicación del teléfono móvil como recurso educacional y formativo. En: AGUIAR, V. (Coord.). CONGRESO EDUTEC-E 2012, XI., 2012, Las Palmas. Las Palmas: Universidad de Las Palmas de Canaria, 2012. p. 1394-1404.

126. BRAZUELO, F. Los teléfonos inteligentes o smartphones en el contexto educativo. En: GALlEGO, D.; ALONSO, C. (Coords.). CONGRESO INTERNACIONAL DE TECNOLOGÍAS PARA LA EDUCACIÓN Y EL CONOCIMIENTO, XVII., 2012, Madrid. UNED. Madrid: Anaya, 2012. ISBN: 978-84-695-3582-0.

127. BRAZUELO, F. Apps o aplicaciones móviles educativas para smartphones y tabletas digitales. En: CACHEIRO, M. L.; GALlEGO, D.; ALONSO, C. (Coords.). CONGRESO INTERNACIONAL DE TECNOLOGÍAS PARA LA EDUCACIÓN Y EL CONOCIMIENTO. INTERESTRATIC, XVIII., 2013, Madrid. UNED. Madrid: Anaya, 2013. ISBN: 978-84-695-7990-9.

128. BRAZUELO, F. Talento emprendedor y aplicaciones móviles. En: BETANCOR, I.; GALLEGO, D.; ALONSO, C. (Coords.). CONGRESO INTERNACIONAL DE GESTIÓN DEL TALENTO, III., 2013, Lanzarote. UNED. Lanzarote: Cabildo de Lanzarote, 2013. ISBN: 978-84-695-7781-3.

129. CÁCERES, M. Enreda City: Vive una nueva realidad. En: GALLEGO, D.; ALONSO, C. (Coords.). CONGRESO INTERNACIONAL DE TECNOLOGÍAS PARA LA EDUCACIÓN Y EL CONOCIMIENTO, XVII., 2012, Madrid. UNED. Madrid: Anaya, 2012. ISBN: 978-84-695-3582-0.

130. CAMUÑAS, N.; LLOPIS, C.; LÓPEZ, E.; LÓPEZ, M.; PASCUAL, D.; ALCAIDE, M. Inclusion of social networks and mobile devices as educational resources in higher education. INTED 2013. Disponible en: < http://library.iated.org/publications/ INTED2013>. Acceso en: 21 dic. 2013. 
131. CAMPO, C.; MANSILLA, J. M. 'Móvil-izados' por las redes sociales. En: GALLEGO, D.; ALONSO, C. (Coords.). CONGRESO INTERNACIONAL DE TECNOLOGÍAS PARA LA EDUCACIÓN Y EL CONOCIMIENTO, XVII., 2012, Madrid. UNED. Madrid: Anaya, 2012. ISBN: 978-84-695-3582-0.

132. CAPDEPÓN, M. Emprediendo con localseo.es: ¿por qué movilizarse? En: GALLEGO, D.; ALONSO, C. (Coords.). CONGRESO INTERNACIONAL DE GESTIÓN DEL TALENTO, III., 2013, Lanzarote. UNED. Lanzarote: Cabildo de Lanzarote, 2013. ISBN: 978-84-695-7781-3.

133. CASCALES, A.; MARTÍNEZ, M. J.; LAGUNA, M. I. Metodología para trabajar con tablets con alumnos de necesidades específicas de apoyo educativo en aulas ordinarias. En: CARDONA, M.; CHINER, E.; GINER, A. (Coords.). INTERNACIONAL MODELOS DE INVESTIGACIÓN EDUCATIVA DE LAASOCIACIÓN INTERUNIVERSITARIA DE INVESTIGACIÓN PEDAGÓGICA, II., 2013, Alicante. Alicante: Asociación Interuniversitaria de Investigación Pedagógica (AIDIPE) y Universidad de Alicante, 2013. p. 718-725.

134. CASTRO, J.; GIMÉNEZ, M.; MONSORIU, J. A. Physics experiments using the mobile acceleration sensor. INTED 2013. Disponible en: $<$ http://library.iated.org/publications/INTED2013>. Acceso en: 21 dic. 2013.

135. GARAIZAR, P.; PEÑA, O.; ROMERO, J. A. Montessori in the mobile era: building new learning experiences through tangible user interfaces. INTED 2013. Disponible en: $<$ http://library.iated.org/publications/INTED2013>. Acceso en: 21 dic. 2013.

136. CARDINALLI, F. Mobile Learning: Myth or Reality? Seminario eMadrid: Oportunidades del Mobile Learning para la Universidad y la empresa. Disponible en: $<$ http:// vimeo.com/25553556. 2011>. Acceso en: 23 dic. $/ 2013$.

137. CONRARI, S.; PÉREZ, R. Cámara digital y teléfono celular como recursos para el estudio cinemático de cuerpos en movimiento. En: GALLEGO, D.; ALONSO, C. (Coords.). CONGRESO INTERNACIONAL DE TECNOLOGÍAS PARA LA EDUCACIÓN Y EL CONOCIMIENTO, XVII., 2012, Madrid. UNED. Madrid: Anaya, 2012. ISBN: 978-84-695-3582-0.

138. CLARES, J.; PERERA, V. Uso de twitter en la formación de maestros. En: FIDALGO, A.; SEIN-ECHALUCE, M. L. (Coords.). CONGRESO INTERNACIONAL SOBRE APRENDIZAJE, INNOVACIÓN Y COMPETIVIDAD, II., 2013, Madrid. Madrid: Fundación General de la Universidad Politécnica de Madrid, 2013. p. 802-805.

139. CHAMORRO, P. Implementation of a peer e-evaluation system in a virtual/mobile learning environment. En: CONGRESO INTERNACIONAL SOBRE INVESTIGACIÓN E INNOVACIÓN EN EDUCACIÓN, IV., 2012. Disponible en: <http://library.iated.org/ view/CHAMORROPOSADA2012IMP>. Acceso en: 23 dic. 2013. 
140. CLARES, J. Uso de los dispositivos móviles en Educación Superior: Twitter. En: CACHEIRO, M. L.; GALLEGO, D.; ALONSO, C. (Coords.). CONGRESO INTERNACIONAL DE TECNOLOGÍAS PARA LA EDUCACIÓN Y EL CONOCIMIENTO. INTERESTRATIC, XVIII., 2013, Madrid. UNED. Madrid: Anaya, 2013. ISBN: 978-84695-7990-9.

141. DE LÁZARO, M., ALCOLEA, M.; PALACIOS, M. Dispositivos móviles: una herramienta para el aprendizaje en Geografía y en los trabajos de campo. En: FIDALGO, A.; SEIN-ECHALUCE, M. L. (Coords.). CONGRESO INTERNACIONAL SOBRE APRENDIZAJE, INNOVACIÓN Y COMPETIVIDAD, II., 2013, Madrid. Madrid: Fundación General de la Universidad Politécnica de Madrid, 2013. p. 685-690.

142. CALVO, R.; MORENO, L.; IGLESIAS, A. Requirements elicitation to design an accessible chat as a synchronous tool in M-learning environments. En: BENGOCHEA, L.; RAMON, J. (Coords.). CONGRESO IBEROAMERICANO SOBRE CALIDAD Y ACCESIBILIDAD DE LA FORMACIÓN VIRTUAL: CAFVIR, III., 201, Madrid. Madrid: Universidad de Alcalá, 2012. p. 357-364.

143. GARCIA, A.; GARCIA, E.; GARCIA-CABOT, A.; BAR-MAGEN, J. Desarrollo de una aplicación para dispositivo móvil para registrar las interacciones del usuario con objetos docentes basados en web. En: BENGOCHEA, L.; RAMON, J. (Coords.). CONGRESO IBEROAMERICANO SOBRE CALIDAD Y ACCESIBILIDAD DE LA FORMACIÓN VIRTUAL: CAFVIR, III., 2012, Madrid. Madrid: Universidad de Alcalá, 2012. p. 177-184.

144. GARCÍA, J.; ALONSO, J. C.; AMESCUA, A. Use of mobile application in spanish universities: present status and future challenges. En: CONGRESO INTERNACIONAL SOBRE INVESTIGACIÓN E INNOVACIÓN EN EDUCACIÓN, IV., 2011. Disponible en: <http://library.iated.org/view/GARCIAGUZMAN2011USE>. Acceso en: 23 dic. 2013.

145. GARCÍA, J. J.; RUÍZ, E.; PALMA, R. Mobile system design for teaching the function concept. En: CONGRESO INTERNACIONAL SOBRE INVESTIGACIÓN E INNOVACIÓN EN EDUCACIÓN, IV., 2013. Disponible en: <http://library.iated.org/ view/GARCIAGUTIERREZ2013MOB>. Acceso en: 23 dic. 2013.

146. GARCÍA, M.; TORO, E.; TOLEDO, C. Laboratorio Móvil Computacional: integración de TIC en el aula. En: GALLEGO, D.; ALONSO, C. (Coords.). CONGRESO INTERNACIONAL DE TECNOLOGÍAS PARA LA EDUCACIÓN Y EL CONOCIMIENTO, XVII., 2012, Madrid. UNED. Madrid: Anaya, 2012. ISBN: 978-84-695-3582-0.

147. GIL, E.; REBAQUE, P.; MINGUILLÓN, J. Mobile learning \& commuting: entrevista contextual y di-seño de escenarios móviles. En: BENGOCHEA, L.; RAMON, J. (Coords.). CONGRESO IBEROAMERICANO SOBRE CALIDAD Y ACCESIBILIDAD DE LA FORMACIÓN VIRTUAL: CAFVIR, III., 2012, Madrid. Madrid: Universidad de Alcalá, 2012. p. 332-340. 
148. COLMEREDO, M.; PEGALAJAR, M. M-Learning en alumnos con necesidades específicas de apoyo educativo: una experiencia con la aplicación PICAA. En: GALLEGO, D.; ALONSO, C. (Coords.). CONGRESO INTERNACIONAL DE TECNOLOGÍAS PARA LA EDUCACIÓN Y EL CONOCIMIENTO, XVII., 2012, Madrid. UNED. Madrid: Anaya, 2012. ISBN: 978-84-695-3582-0.

149. GÓMEZ, M.; ROCA, C.; SANJUÁN, A.; ROMERO, J.; MARTÍN. J. Realidad Virtual vs Realidad Aumentada vs PDF3D como herramienta de entrenamiento para la mejora de las habilidades espaciales y de los resultados académicos de los alumnos de ingeniería gráfica. En: FIDALGO, A.; SEIN-ECHALUCE, M. L. (Coords.). CONGRESO INTERNACIONAL SOBRE APRENDIZAJE, INNOVACIÓN Y COMPETITIVIDAD, II., 2013, Madrid. Madrid: Fundación General de la Universidad Politécnica de Madrid, 2013. p. 47-52.

150. GÓMEZ, M.; BONNIN, J. Uso de la Realidad Aumentada Móvil en un Campus Universitario. En: GALLEGO, D.; ALONSO, C. (Coords.). CONGRESO INTERNACIONAL DE TECNOLOGÍAS PARA LA EDUCACIÓN Y EL CONOCIMIENTO, XVI., 2011, Madrid. UNED. Madrid: Anaya, 2011. ISBN: 978-972-8939-45-8.

151. GÓMEZ, M.; GARCÍA, B.; MORENO, F. Dispositivos móviles en red para educación y formación. En: GALLEGO, D.; ALONSO, C. (Coords.). CONGRESO INTERNACIONAL DE TECNOLOGÍAS PARA LA EDUCACIÓN Y EL CONOCIMIENTO, XVI., 2011, Madrid. UNED. Madrid: Anaya, 2011. ISBN: 978-972-8939-45-8.

152. GÓMEZ, M.; IZQUIERDO, A.; FERNÁNDEZ, D. Tablet PC, bilingüismo y curriculum integrado. En: GALLEGO, D.; ALONSO, C. (Coords.). CONGRESO INTERNACIONAL DE TECNOLOGÍAS PARA LA EDUCACIÓN Y EL CONOCIMIENTO, XVI., 2011, Madrid. UNED. Madrid: Anaya, 2011. ISBN: 978-972-8939-45-8.

153. GÓMEZ, M. eDUMOVIL: Educación y dispositivos móviles para aprendizaje en red. En: GALLEGO, D.; ALONSO, C. (Coords.). CONGRESO INTERNACIONAL DE TECNOLOGÍAS PARA LA EDUCACIÓN Y EL CONOCIMIENTO, XVII., 2012, Madrid. UNED. Madrid: Anaya, 2012. ISBN: 978-84-695-3582-0.

154. GÓMEZ, M. Educación Aumentada: movilidad y tecnología de posición para educación. En: CACHEIRO, M. L.; GALlEGO, D.; ALONSO, C. (Coords.). CONGRESO INTERNACIONAL DE TECNOLOGÍAS PARA LA EDUCACIÓN Y EL CONOCIMIENTO. INTERESTRATIC, XVIII., 2013, Madrid. UNED. Madrid: Anaya, 2013. ISBN: 978-84-695-7990-9.

155. GÓMEZ, M. Educación aumentada con Realidad Aumentada. La tecnología móvil se integra en el entorno de aprendizaje. En: CONGRESO INTERNACIONAL BUENAS PRÁCTICAS CON TIC UNIVERSIDAD DE MÁLAGA, III., 2013. Disponible en: $<$ https://www.youtube.com/watch?v=tZ5_c3t8o-c>. Acceso en: 21 dic. 2013.

156. GONZÁLEZ, D. Experiencia de innovación docente mediante el empleo de objetos de aprendizaje de materiales de construcción en un escenario educativo de Mobile Lear- 
ning. En: CUÉLLAR, M. J.; O’DWYER, J.; MARRERO, H. (Coords.). JORNADAS DE INNOVACIÓN EDUCATIVA DE LA ULL, III., Tenerife, 2013. Tenerife: Universidad de La Laguna, 2013. p. 108-120.

157. GONZÁLEZ, N. El m-learning en el aprendizaje presencial: un curso híbrido en economía. En: FIDALGO, A.; SEIN-ECHALUCE, M. L. (Coords.). CONGRESO INTERNACIONAL SOBRE APRENDIZAJE, INNOVACIÓN Y COMPETIVIDAD, II., 2013, Madrid. Madrid: Fundación General de la Universidad Politécnica de Madrid, 2013. p. 697-702.

158. LIJÓ, J. A. La Realidad Aumentada en la Educación, sus aplicaciones en la asignatura de Educación Plástica y Visual en ESO. En: GALlEGO, D.; ALONSO, C. (Coords.). CONGRESO INTERNACIONAL DE TECNOLOGÍAS PARA LA EDUCACIÓN Y EL CONOCIMIENTO, XVII., 2012, Madrid. UNED. Madrid: Anaya, 2012. ISBN: 978-84-695-3582-0.

159. LÓPEZ, A. Identificación y análisis de los patrones de uso de los teléfonos smartphone en la población universitaria de la UPV-EHU. En: CONGRESO INTERNACIONAL DE EDUCACIÓN ABIERTA Y TECNOLOGÍA. Ikasnabar, VI., 2013. Disponible en: $<$ http://ikasnabar.com/papers>. Acceso en: 20 dic. 2013.

160. MAGDALENA, T.; FERREIRA, S. M-Learning: Programa de Capacitación a Distancia para la Enseñanza del Español en Brasil en un contexto intercultural Brasil-España. En: GALLEGO, D.; ALONSO, C. (Coords.). CONGRESO INTERNACIONAL DE TECNOLOGÍAS PARA LA EDUCACIÓN Y EL CONOCIMIENTO, XVII., 2012, Madrid. UNED. Madrid: Anaya, 2012. ISBN: 978-84-695-3582-0.

161. MAGAL-ROYO, T.; GIMÉNEZ, J. L.; GARCÍA, J. El aprendizaje y las tecnologías ubicuas en el entorno universitario. En: GALLEGO, D.; ALONSO, C. (Coords.). CONGRESO INTERNACIONAL DE TECNOLOGÍAS PARA LA EDUCACIÓN Y EL CONOCIMIENTO, XVII., 2012, Madrid. UNED. Madrid: Anaya, 2012. ISBN: 978-84-695-3582-0.

162. MARQUÉS, P. Claves para mejorar los aprendizajes integrando las tecnologías móviles en las clases. En: NAVARIDAS, F.; SANTIAGO, R.; SOTA 1, J.; JIMÉNEZ, M. A.; GONZÁLEZ, L. (Coords.). Jornadas sobre tecnología móvil e innovación en el aula. Logroño, España, 2013.

163. MARTÍN, V.; SOTO, O.; ESCARBAJAL, A.; SOTO, J. Material didáctico digital en formato de libro de texto interactivo: iBooks Author. En: CUÉLLAR, M. J.; O'DWYER, J.; MARRERO, H. (Coords.). JORNADAS DE INNOVACIÓN EDUCATIVA DE LA ULL, III., 2013, Tenerife. Tenerife: Universidad de La Laguna, 2013. p. 185-195.

164. MATOS, J. C.; PÉREZ, M., GONZÁLEZ, B.; ESCUADRA, J. U-learning y autoaprendizaje activo. En: PÉREZ, J. L. (Coord.). Jornadas de Innovación Educativa: El Espacio Europeo de Educación Superior Zamora: Universidad de Zamora, 2012. p. 83-86. 
165. MONTOLÍO, M. De la pizarra tradicional a la tableta digital. En: GALLEGO, D.; ALONSO, C. (Coords.). CONGRESO INTERNACIONAL DE TECNOLOGÍAS PARA LA EDUCACIÓN Y EL CONOCIMIENTO, XVII., 2012, Madrid. UNED. Madrid: Anaya, 2012. ISBN: 978-84-695-3582-0.

166. MORALES, P. La tableta digital y su uso en educación. Una experiencia en educación de adultos. En: GALLEGO, D.; ALONSO, C. (Coords.). CONGRESO INTERNACIONAL DE TECNOLOGÍAS PARA LA EDUCACIÓN Y EL CONOCIMIENTO, XVII., UNED. Madrid: Anaya, 2012. ISBN: 978-84-695-3582-0.

167. MUÑOZ, M.; AGUADED, I. La competencia digital en el alumnado con necesidades especiales. Uso de ordenadores, tabletas, internet, e-books y narraciones digitales en el aula. En: CONGRESO INTERNACIONAL INNOVAGOGÍA, I., 2012. Disponible en: <http://riemann.upo.es/congresos/index.php/innovagogia2012/Iinnovagogia2012/ schedConf/presentations>. Acceso en: 21 dic. 2013.

168. OLMEDO, K.; GRANÉ. M.; SUÁREZ, R. Aprendizaje móvil... Nuevas herramientas, ¿Nuevas estrategias? En: AGUIAR, V. (Coord.). CONGRESO EDUTEC-E 2012, XI., 2012, Las Palmas. Las Palmas: Universidad de Las Palmas de Canaria, 2012. p. 1265-1272.

169.OLMEDO, K.; GRANÉ, M.; CRECENZI, L.; SUÁREZ, R. Dispositivos móviles y su integración en entornos e-learning tradicionales. En: SÁNCHEZ, J.; FRAGA, L.; ARRAZOLA, J.; MIÑO, R.; GIRÓ, X. (Coords.). EUROPEAN CONFERENCE ON INFORMATION TECHNOLOGY IN EDUCATION AND SOCIETY: A CRITICAL INSIGHT, III., 2012, Barcelona. Barcelona: Universidad de Barcelona, 2012. p. 215-217.

170. PEREIRA, N.; FERREIRA, S. Proceso de aprendizaje asistido por tecnologías virtuales. En: GÉRTRUDIX, M.; GÉRTRUDIX, F. (Coords.). CONGRESO INTERNACIONAL SOCIEDAD DIGITAL: ESPACIOS PARA LA INTERACTIVIDAD Y LA INMERSIÓN, II., 2011, Madrid. Madrid: Universidad Complutense de Madrid, 2011. p. 314-321.

171. POYATOS, C. Gymkanas educativas con códigos QR. En: GALLEGO, D.; ALONSO, C. (Coords.). CONGRESO INTERNACIONAL DE TECNOLOGÍAS PARA LA EDUCACIÓN Y EL CONOCIMIENTO, XVII., 2012, Madrid. UNED. Madrid: Anaya, 2012. ISBN: 978-84-695-3582-0.

172. PUCHEU, D. L'informatique ubiquitaire. En: GALLEGO, D.; ALONSO, C. (Coords.). CONGRESO INTERNACIONAL DE TECNOLOGÍAS PARA LA EDUCACIÓN Y EL CONOCIMIENTO, XVII., 2012, Madrid. UNED. Madrid: Anaya, 2012. ISBN: 978-84-695-3582-0.

173. RAMÍREZ, M. Inclusión de mobile learning en ambientes virtuales de aprendizaje. En: GARCÍA-VALCÁRCEL, A. (Coord.). CONGRESO INTERNACIONAL DE INTERCAMBIO DE EXPERIENCIAS DE INNOVACIÓN DOCENTE UNIVERSITARIA, I., 2012, Cartagena. Cartagena: Universidad Politécnica de Cartagena y Universidad de Murcia, 2012. p. 71-88. 
174. ROBLES, G. Una experiencia de m-learning: gymkhanas con smartphones. En: SEMINARIO EMADRID SOBRE “JUEGOS, APRENDIZAJE Y MOVILIDAD”, 2010. Acceso en: 23 dic. 2013. Disponible en: <http://vimeo.com/17001912>.

175. ROMÁN, P.; DÍAZ, M.; AMUEDO, A.; SUBIRA, I. Nuevos grados de conocimiento y uso de la telefonía móvil y herramientas web 2.0 en la educación con estudiantes universitarios de Sevilla y Córdoba. En: AGUIAR, V. (Coord.). CONGRESO EDUTEC-E 2012, XI., 2012, Las Palmas. Las Palmas: Universidad de Las Palmas de Canaria, 2012. p. 529-543.

176. ROMERO, S.; PAMPLONA, S.; PEREZ, M. J.; MONFERRER, J. La implantación del Tablet Pc en el proceso de aprendizaje a distancia. En: BENGOCHEA, L.; RAMON, J. (Coords.). CONGRESO IBEROAMERICANO SOBRE CALIDAD Y ACCESIBILIDAD DE LA FORMACIÓN VIRTUAL: CAFVIR, III., 2012, Madrid. Madrid: Universidad de Alcalá. 2012, p. 349-356.

177. ROMERO, R.; ZARRAONANDIA, T.; AEDO, I.; DÍAZ, P. Diseñando material educativo usable para cursos de inglés soportados mediante dispositivos móviles. En: GALLEGO, D.; ALONSO, C. (Coords.). CONGRESO INTERNACIONAL DE TECNOLOGÍAS PARA LA EDUCACIÓN Y EL CONOCIMIENTO, XV., 2010, Madrid. UNED. Madrid: Anaya, 2010. ISBN: 978-84-693-2709-8.

178. SAGAR. C. Mobile Learning and learning english as a foreign language for adults: challenges and strategies for future mobile learning projects. INTED 2013. Disponible en: <http://library.iated.org/publications/INTED2013>. Acceso en: 21 dic. 2013

179. SAMINO, R.; MANERA, J.; RODRÍGUEZ, J.; RIVERO, L. El podcast adaptado al entorno educativo como recurso innovador para el aprendizaje. En: GARCÍA, J. J. (Coord.). CONGRESO ANUAL AEDEM, XXVII., 2013, Huelva. Huelva: Universidad de Huelva, 2013.

180. SOUZA, M; FERREIRA, S. Microcontenidos educativos para el aprendizaje móvil. En: GÉRTRUDIX, M.; GÉRTRUDIX, F. (Coords.). CONGRESO INTERNACIONAL SOCIEDAD DIGITAL: ESPACIOS PARA LA INTERACTIVIDAD Y LA INMERSIÓN, II., 2011, Madrid. Madrid: Universidad Complutense de Madrid, 2011. p. 1197-1206.

181. STANO, E.; SANCHO, J.; GRANÉ, M.; PAULA, I.; SUÁREZ, R. Uso de dispositivos móviles en ámbitos educativos no formales para colectivos con necesidades específicas. En: AGUIAR, V. (Coord.). CONGRESO EDUTEC-E 2012, XI., 2012, Las Palmas. Las Palmas: Universidad de Las Palmas de Canaria, 2012. p. 595-603.

182. TORRENTE, J. Desarrollo de juegos digitales educativos para dispositivos móviles: el proyecto para Android. En: SEMINARIO EMADRID SOBRE “JUEGOS, APRENDIZAJE Y MOVILIDAD”. 2010. Disponible en: <http://vimeo.com/17002066>. Acceso en: 23 dic. 2013.

183. UBEDA, J. Smartphones, marketing y educación: taxonomía e implicaciones de marketing a partir de un análisis de aplicaciones móviles de universidades españolas y de 
escala mundial. En: GARCÍA, J. J. (Coord.). CONGRESO ANUAL AEDEM, XXVII., 2013, Huelva. Huelva: Universidad de Huelva, 2013.

184. VALVERDE, J. C.; MARTÍNEZ, S. A mobile assisted blended-learning experience. En: INTERNATIONAL CONFERENCE ON EDUCATION AND NEW LEARNING TECHNOLOGIES (EDULEARN 2012), 2012. Disponible en: < http://library.iated.org/ view/VALVERDE2012AMO>. Acceso en: 22 dic. 2013.

185. VÁZQUEZ, R.; ANGULO, F. Learning in any place: the use of mobile technologies. INTED 2010. Disponible en: <http://library.iated.org/view/VAZQUEZ2010LEA>. Acceso en: 21 dic. 2013.

186. VIDAL, A. M-learning y enseñanza universitaria de lenguas para la paz. En: AGUIAR, V. (Coord.). CONGRESO EDUTEC-E 2012, XI., 2012, Las Palmas. Las Palmas: Universidad de Las Palmas de Canaria, 2012. p. 982-992.

187. ZAMORA, J. L.; BELLO, S. Nuevas Tecnologías aplicadas a la docencia universitaria en el ámbito del derecho mediante códigos bidimensionales. En: GALLEGO, D.; ALONSO, C. (Coords.). CONGRESO INTERNACIONAL DE TECNOLOGÍAS PARA LA EDUCACIÓN Y EL CONOCIMIENTO, XVII., 2012. Madrid. UNED. Madrid: Anaya, 2012. ISBN: 978-84-695-3582-0.

188. ZAMORA, J.; BELLO, S. Codificación y mobile tagging como herramientas tecnológicas aplicadas a la enseñanza: bidiblogs y videocreatividad. En: AGUIAR, V. (Coord.). CONGRESO EDUTEC-E 2012, XI., 2012, Las Palmas. Las Palmas: Universidad de Las Palmas de Canaria, 2012. p. 1147-1152.

189. ZAMORA, J.; BELLO, S. Uso de aplicaciones para dispositivos móviles como herramienta de aprendizaje en el mundo del Derecho. En: CACHEIRO, M. L.; GALLEGO, D.; ALONSO, C. (Coords.). CONGRESO INTERNACIONAL DE TECNOLOGÍAS PARA LA EDUCACIÓN Y EL CONOCIMIENTO. INTERESTRATIC, XVIII., 2013, Madrid. UNED. Madrid: Anaya, 2013. ISBN: 978-84-695-7990-9.

190. ZAPATA, M. Calidad centrada en el aprendizaje en sistemas de gestión que integren tecnología móvil y software social. En: BENGOCHEA, L.; RAMON, J. (Coords.). CONGRESO IBEROAMERICANO SOBRE CALIDAD Y ACCESIBILIDAD DE LA FORMACIÓN VIRTUAL: CAFVIR, III., 2012, Madrid. Madrid: Universidad de Alcalá, 2012. p. 457-467.

Texto recebido em 21 de novembro de 2014. Texto aprovado em 09 de dezembro de 2014. 